\title{
Correction to: Circular Economy Business Models and Practices
}

\author{
Anna Itkin
}

Correction to:

Chapter "Circular Economy Business Models

and Practices" in: L. Liu and S. Ramakrishna (eds.),

An Introduction to Circular Economy, https://doi.org/10.1007/978-981-15-8510-4_22

The original version of the book was inadvertently published with incorrect figure captions and without figure numbers in Chapter 22. Figure captions 1, 4 and 5 have been replaced with the correct figure captions and numbers in the updated version. Also, author bio and photo has included in the updated version. 